\title{
Growth Kinetics of Core-Shell Au/Ag Nanoparticles
}

\author{
Cyrille Hamon* and Doru Constantin* \\ Université Paris-Saclay, CNRS, Laboratoire de Physique des Solides, 91405, Orsay, France.
}

E-mail: cyrille.hamon@u-psud.fr; doru.constantin@u-psud.fr 


\begin{abstract}
We study by synchrotron-based time-resolved small angle X-ray scattering the growth of silver shells on gold nanorods in the presence of hexadecyltrimethylammonium chloride (CTAC) as a function of the silver/gold molar ratio $K$. For all but the highest $K$ values, the silver precursor is completely converted to the solid phase. The growth rate of the width decreases exponentially, and its starting value is linear in $K$, with a slope of $10.3 \pm 0.2 \mathrm{~nm} / \mathrm{s} / \mathrm{M}$. A simple growth model including these features accounts for the magnitude of the evolution time. Assuming that permeation across the CTAC bilayer is the rate-limiting step we obtain a permeability of $\simeq 1 \mu \mathrm{m} / \mathrm{s}$.
\end{abstract}

\title{
Introduction
}

Bimetallic particles (BPs) consisting of a gold core covered with a silver shell ${ }^{1,2}$ are very promising in view of applications, as they allow fine control over the position of the plasmon resonance peaks. Their growth has been monitored over time using UV-Vis-NIR absorbance spectroscopy (AS). ${ }^{3-5}$ This technique is widely available, has excellent time resolution and is very sensitive to the particle morphology, but the position of the plasmon peak cannot be used to directly retrieve particle dimensions in the case of anisotropic nanoparticles; thus, one cannot extract the complete kinetical information using AS. Promising results have recently been obtained using environmental transmission electron microscopy, ${ }^{6,7}$ but care must be taken in interpreting them because of the pronounced effect of the electron beam on the growth process.

We performed time-resolved small-angle X-ray scattering studies (SAXS) at a highbrilliance synchrotron beamline on BP suspensions. This technique has been extensively used to characterize the growth of homogeneous (single-metal) spherical ${ }^{8-13}$ and anisotropic ${ }^{14-16}$ nanoparticles but it has been very seldom applied to bimetallic objects, in particular because the data treatment is much more involved in this case. The advantage of SAXS is that it yields the particle size unambiguously. 
Aside from the interest in the optical properties of BPs, studying their formation kinetics can also answer fundamental questions related to metal growth at the nanoscale: the use of monodisperse seeds helps maintain a uniform particle population, while varying the seed morphology gives control over the properties of the shell: isotropic or elongated, mono- or polycrystalline, and so on. Furthermore, changing the reaction conditions can provide insight into the role of various additives, as we have shown recently for the dual action of ascorbic acid in the growth of silver onto gold bipyramids. ${ }^{7}$

Single-crystal gold nanorods (AuNRs) exhibit a transverse octogonal cross section enclosed by $\{520\}$ facets and terminated by a combination of $\{110\}$ and $\{111\}$ facets at the tips. ${ }^{17,18}$ After silver coating, the resulting BPs adopt a cuboidal shape enclosed by six $\{100\}$ facets. ${ }^{3,4,19}$ In a previous work, we systematically tuned the cross section of the BPs by varying the silver/gold molar ratio $K$ in the synthesis and self-assembled the nanoparticles into

supercrystals. ${ }^{20}$ We found that the initial hexagonal structure of the supercrystal shifts to a square in-plane arrangement at low $K$ values, suggesting that the BPs already adopt a cuboidal morphology for a very thin silver shell (a few nm). Motivated by these findings, we monitored the shape evolution of the AuNRs upon silver coating by SAXS to retrieve the growth rate as a function of the $K$ value.

\section{Methods}

\section{Particle Synthesis}

Materials: All of the reactants were purchased from Sigma-Aldrich and used without further purification: hexadecyltrimethylammonium bromide (CTAB, $\geq 99 \%$ ), hexadecyltrimethylammonium chloride (CTAC, $25 \mathrm{wt} \%$ in H2O), 5-bromosalicylic acid (90\%), hydrogen tetrachloroaurate trihydrate $\left(\mathrm{HAuCl}_{4} \cdot 3 \mathrm{H}_{2} \mathrm{O}, \geq 99.9 \%\right)$, silver nitrate $\left(\mathrm{AgNO}_{3}, \geq 99 \%\right)$, L-ascorbic acid $(\mathrm{AA}, \geq 99 \%)$, and sodium borohydride $\left(\mathrm{NaBH}_{4}, 99 \%\right)$. Water purified by reverse osmosis with a resistivity ( $>15 \mathrm{M} \Omega . \mathrm{cm})$ was used in all experiments. 
Gold Nanorod Synthesis: AuNRs were synthesized through a seed-mediated approach according to previously reported methods. ${ }^{21,22}$ For the preparation of the seeds, $50 \mu \mathrm{L}$ of a $0.025 \mathrm{M} \mathrm{HAuCl}_{4}$ solution was added to $4.7 \mathrm{~mL}$ of $0.1 \mathrm{M} \mathrm{CTAB}$ solution at $30{ }^{\circ} \mathrm{C}$; after mixing, $300 \mu \mathrm{L}$ of a freshly prepared $0.01 \mathrm{M} \mathrm{NaBH}_{4}$ solution was injected under vigorous stirring. The growth solution was constituted with $100 \mathrm{~mL}$ of $0.05 \mathrm{M}$ CTAB, in which $90 \mathrm{mg}$ of 5-bromosalicylic acid were dissolved. Then, $960 \mu \mathrm{L}$ of $0.01 \mathrm{M} \mathrm{AgNO}_{3}$ and $2 \mathrm{~mL}$ of $0.025 \mathrm{M} \mathrm{HAuCl}_{4}$ solution were added to the mixture. The absorbance at $396 \mathrm{~nm}$ was monitored in a cuvette with $1 \mathrm{~cm}$ optical path length until it reached 0.65 , indicating suitable prereduction conditions. Then $260 \mu \mathrm{L}$ of $0.1 \mathrm{M}$ AA solution was added under vigorous stirring, immediately followed by $160 \mu \mathrm{L}$ of seed solution. The mixture was left undisturbed at $30{ }^{\circ} \mathrm{C}$ for at least $4 \mathrm{~h}$. Gold nanorods presented an LSPR with an absorption maximum at $720 \mathrm{~nm}$. The suspension was purified by three centrifugation steps (at $7100 \mathrm{~g}$, for $40 \mathrm{~min}$ ) and pellet redispersion in a solution of $1 \mathrm{mM}$ CTAC. AuNR suspensions were stable for months.

Silver Shell Growth: Overgrowth was performed according to recently published protocols. ${ }^{5,19}$ The purified AuNR were centrifuged at $8500 \mathrm{~g}$ for 30 min and redispersed in a $10 \mathrm{mM} \mathrm{CTAC}$ solution at a final gold concentration of $0.25 \mathrm{mM}$ in all samples. Silver and ascorbic acid were added in sequence to avoid silver particle formation. The molar ratio between the AA and the Ag precursor was fixed to 4 in all experiments. The silver-to-gold molar ratio $K$ (Ag ions per Au atoms in the nanorods, at the beginning of the process) was varied between 0.4 and 16. For instance, in the case of $K=2,50 \mu \mathrm{L}$ of a $0.1 \mathrm{M} \mathrm{AgNO}_{3}$ solution was injected in $10 \mathrm{~mL}$ of AuNR suspension. After brief shaking, the solution was supplemented with $50 \mu \mathrm{L}$ of a $0.4 \mathrm{M}$ AA solution and shaken again. 


\section{TR-SAXS}

SAXS measurements were performed on the SWING beamline of the SOLEIL synchrotron (Saint-Aubin, France). The sample-to-detector distance was $6.52 \mathrm{~m}$ and the beam energy $E=12 \mathrm{keV}$, covering a scattering vector range $0.0015<q<0.2 \AA^{-1}$. The samples were contained in cylindrical glass capillaries (Mark-Rörchen, Germany) of calibrated diameter, placed vertically in a motorized and temperature-controlled holder. The measurements were performed at room temperature $\left(22{ }^{\circ} \mathrm{C}\right)$ and at $60{ }^{\circ} \mathrm{C}$. The beam size was approximately $500 \times 200 \mu \mathrm{m}^{2}$ (horiz. $\times$ vert.)

The scattered signal was recorded by an Eiger 4M detector (Dectris Ltd., Switzerland) with pixel size $75 \mu \mathrm{m}$. Preliminary data treatment (angular averaging and normalization) was done using software Foxtrot developed at the beamline and yielded the intensity as a function of the scattering vector $I(q)$ in absolute units. Subsequent data modeling was done in Igor Pro using some models available in the NCNR SANS package ${ }^{23}$ and others developed in-house.

\section{Results}

\section{Core Particles}

The scattering signal of the core AuNRs (bottom curve in the left panel of Figure 1) is well described by a polydisperse spherocylinder model with radius $R=52 \AA$ and total length $H+2 R=418 \AA$, where $H=314 \AA$ is the length of the cylindrical mid-section. The (homothetical) size polydispersity $p=0.15$.

\section{Time Evolution}

The signal acquired during the growth kinetics (shown in Figure 1 for two Ag concentrations) is modeled by a core-shell geometry, where the Au core corresponds to the initial particles 

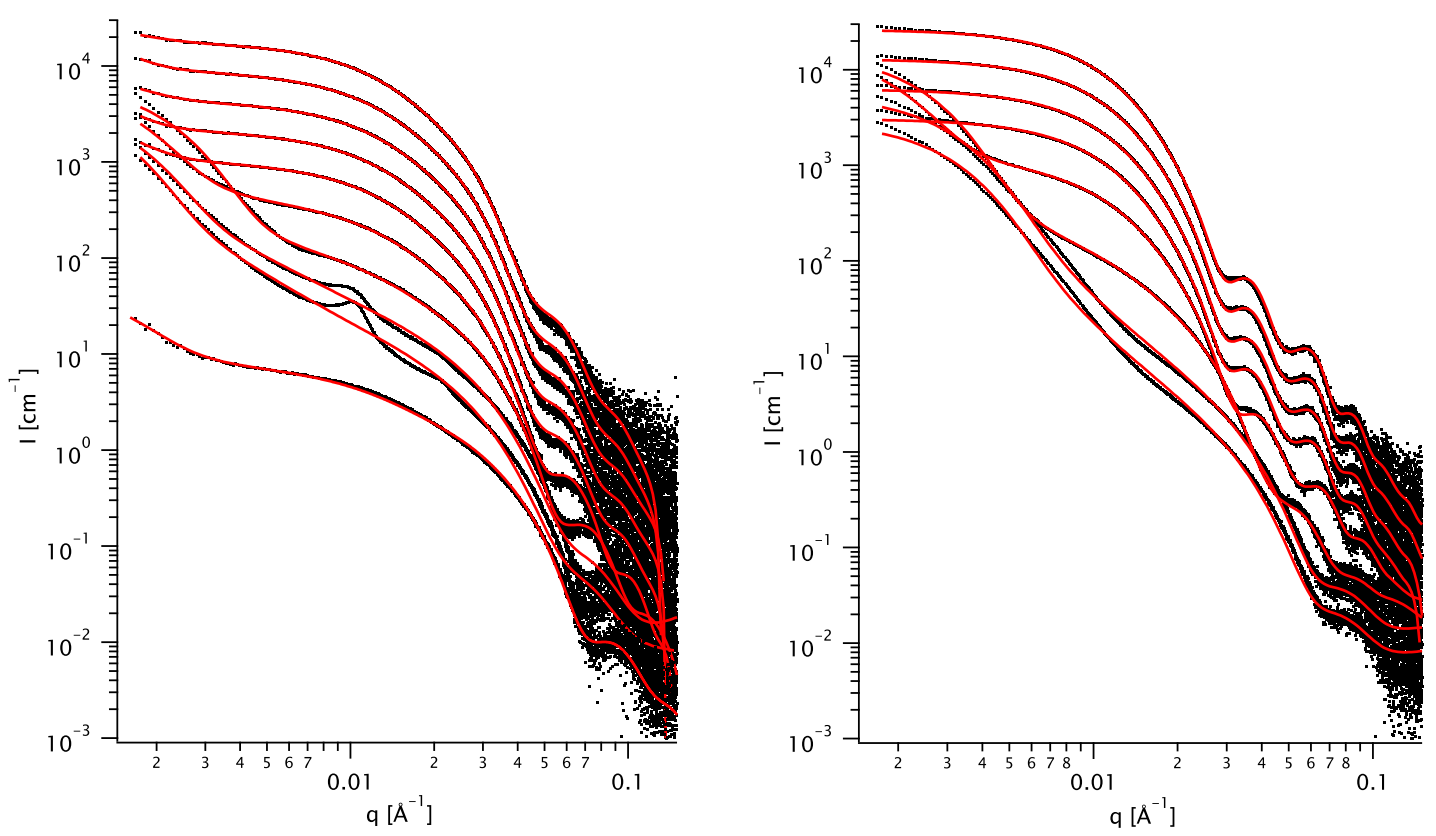

Figure 1: SAXS signal of the nanoparticles at various times during the synthesis (dots), with full-curve fits (lines), for $\mathrm{Ag} / \mathrm{Au}$ ratios $K=2$ (left) and 8 (right). For clarity, successive curves are shifted vertically by factors of 2 (the time increases upwards). The bottom curve in the left panel is the signal of AuNRs before the addition of silver.

and the Ag shell is a prism of width $W$ and length $L$. A (homothetical) size polydispersity $p$ is also accounted for. The details are given in the Supporting Information. One important detail is that, for simplicity, the spherocylinders are modeled as simple cylinders of length $H+R=366 \AA$ (empirically, this value yields a signal very close to that of the full particle.) For consistency, the minimum value of $L$ is fixed at this truncated value, rather than the full $H+2 R$.

The time-dependent width $W(t)$ is shown as symbols in Figure 2 (left), as well as the fit (dashed lines) with the model:

$$
W(t)=2 R+\left(W_{f}-2 R\right)[1-\exp (-t / \tau)]
$$

The fit parameters $W_{f}$ (final width) and $\tau$ (characteristic time) are shown in Figure 2 (right).

At high enough Ag concentration, the length also behaves exponentially, similarly to Eq. (1), see Figure 3. At lower concentration, there is no length increase. 
At short times, the $I(q)$ curves exhibit an increase at small angles, that we model by a Voigt function, and sometimes (as at the bottom left of Figure 1) small structure peaks. Both these features disappear in time. We attribute them to the presence of large aggregates of silver precursor and to transient aggregation of the gold cores, respectively.
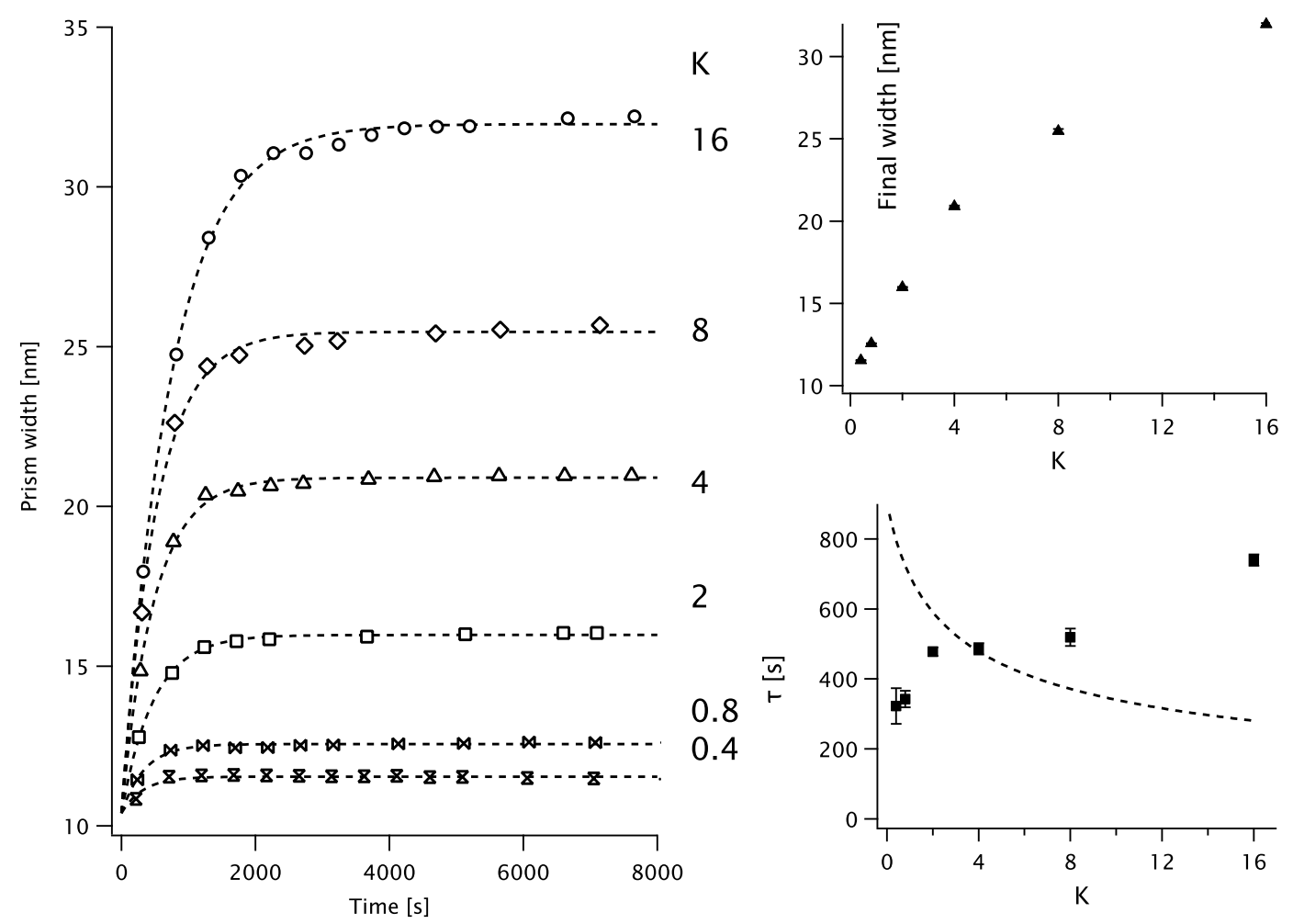

Figure 2: Left: BP width $W$ as a function of time (various symbols) with exponential fits (dashed lines) for the $\mathrm{Ag} / \mathrm{Au}$ ratios $K$ indicated alongside the curves. Right: Fit parameters (symbolss) as a function of $K$ : final width $W_{f}$ (top) and typical time $\tau$ (bottom) with the model (3) (dashed line).

\section{Discussion and Conclusions}

The number concentration of particles is the same for all samples: $n_{\text {part }}=5.610^{17}$ part $/ \mathrm{m}^{3}$ (SAXS) or $7.810^{17}$ part $/ \mathrm{m}^{3}$ (AS), amounting to about one particle per cubic micrometer. The diffusion constants of the ions being of the order of $10^{-9} \mathrm{~m}^{2} / \mathrm{s}$ and that of surfactant micelles about 10 times lower, the concentrations equilibrate over the typical distance $d_{\text {part }}=n_{\text {part }}^{-1 / 3}$ 


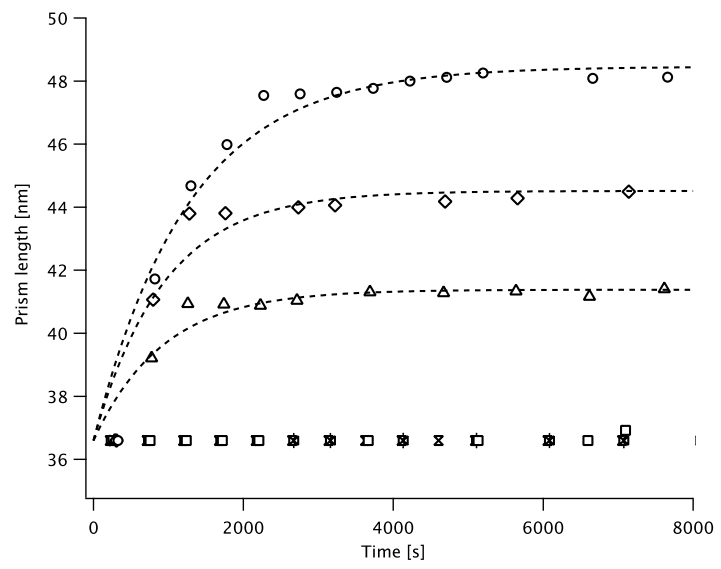

Figure 3: BP length $L$ as a function of time (various symbols) and exponential fits (dashed lines) for $K=4,8$, and 16 .

in milliseconds, much lower than the typical reactions times of a few minutes. The kinetics is thus driven by interface reaction processes, not by diffusion.

Once all of the geometrical parameters are known, we can calculate the volume of the Ag shell and the molar $\mathrm{Ag} / \mathrm{Au}$ ratio within the particles. This parameter is shown for various initial $\mathrm{Ag} / \mathrm{Au}$ ratios in solution $K$ in Figure (4). At long times, the particle composition is very close to $K$, showing that all of the Ag precursor has been converted to the solid phase (except for the highest value $K=16$ ).

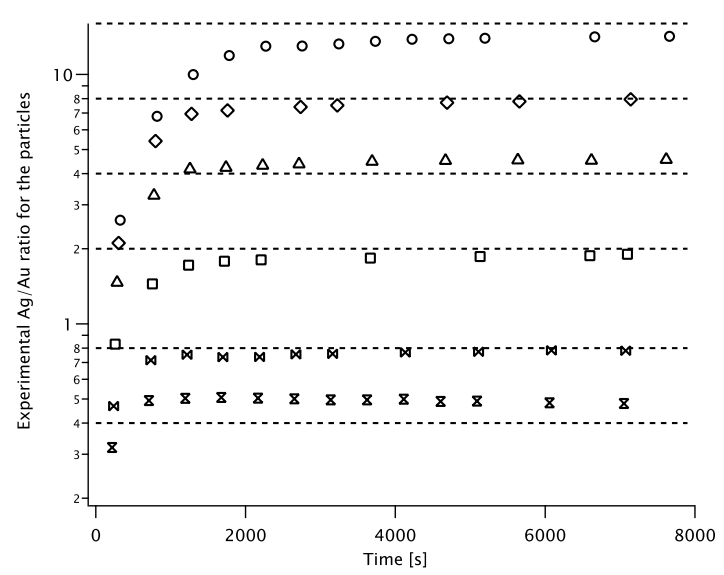

Figure 4: Particle composition: molar $\mathrm{Ag} / \mathrm{Au}$ ratio as a function of time (various symbols) for $\mathrm{Ag} / \mathrm{Au}$ ratios in solution $K=0.4,0.8,24,8$ and 16 (shown as dashed lines).

From (1) we can extract the initial interface velocity along the width $u(0)=\frac{\mathrm{d} W}{\mathrm{~d} t}=\frac{W_{f}-2 R}{2 \tau}$ and along the length $v(0)$. These values are shown in Figure 5. 


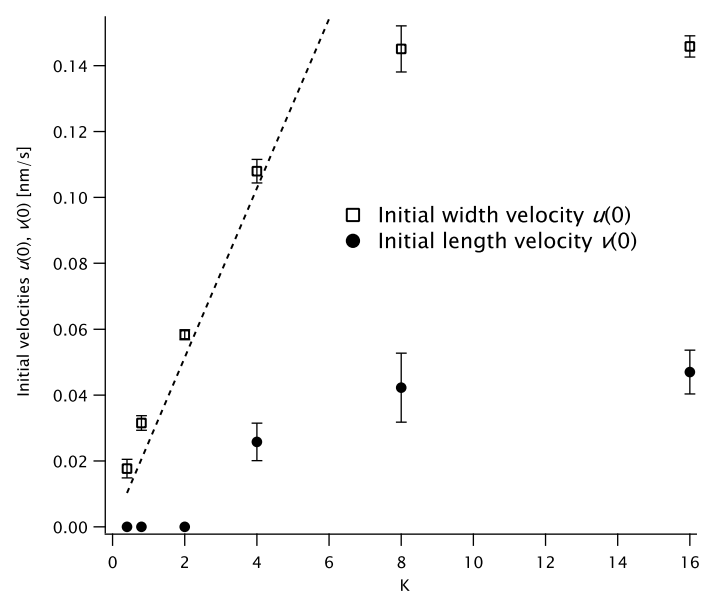

Figure 5: Initial width (squares) and length (solid dots) velocities $u(0)$ and $v(0)$ as a function of $K$. The linear fit to the width velocity (up to $K=8$ ) is shown as dashed line.

Although both the side and tip facets belong to the $\{100\}$ family, longitudinal growth is limited. ${ }^{5,20}$ In the following, we will focus on the width evolution.

For moderate $\mathrm{Ag} / \mathrm{Au}$ ratios (at least up to $K=4$ ), the initial velocity $u(0)$ is linear in $K$, and hence in the molar $\mathrm{Ag}$ concentration $C_{\mathrm{Ag}}$ in the solution at the beginning of the process. We will assume that this dependence holds during growth, yielding a very simple model of the Berthoud type ${ }^{24,25}$ in the limit of vanishing Damköhler number

$$
u(t)=\alpha\left[C_{\mathrm{Ag}}(t)-C_{S}\right]
$$

where the interface attachment rate is $\alpha=10.3 \pm 0.2 \mathrm{~nm} / \mathrm{s} / \mathrm{M}$ and, because all of the silver is finally converted, we set the saturation concentration $C_{S}$ to zero.

Let us now discuss the relaxation time $\tau$ and its dependence on $K$. The model presented in the Supporting Information yields:

$$
\tau=\frac{1}{\gamma K} \frac{\sqrt{\pi} R}{2 \alpha C_{\mathrm{Au}}}
$$

where $\gamma$ is a dimensionless parameter that depends on $K$ and is obtained by fitting an exponential function to the numerical solution of the model as detailed in the Supporting Information. The prediction (3) is plotted and shown in Figure 2 alongside the experimental 
data for $\tau$. The latter are more or less constant (if we ignore the point $K=16$, which is also anomalous with respect to $u(0))$, with a possible decrease as $K$ goes to zero. In contrast, our model $\tau(K)$ increases markedly close to the origin. We have no explanation for this discrepancy. To conclude, our results are well described by the exponential model (1). The interface velocity (and hence the flux of silver ions) are linear in the precursor concentration $C_{\mathrm{Ag}}$ (indicating a Fick-type mechanism) and decrease with time as the precursor is consumed. This behavior is in contrast with the results of Ref. 5, where the kinetics is sigmoidal, with a growth rate that increases linearly, reaches a plateau and then decreases suddenly.

Our model for the growth kinetics captures the magnitude of the typical evolution time $\tau$ but not its variation with $K$.

As defined in Equation (2), $\alpha$ is an overall coefficient, with no assumption concerning the detailed attachment mechanism. Previous studies of the same system highlighted the role of the surfactant bilayer: replacing the CTAC with benzylhexadecyldimethylammonium chloride (BDAC) slows down the process by one order of magnitude. ${ }^{5}$

Assuming that bilayer crossing is the rate-limiting step, we can define a permeability $P=\alpha \frac{\rho_{\mathrm{Ag}}}{M_{\mathrm{Ag}}} \simeq 10^{-6} \mathrm{~m} / \mathrm{s}$. This value can be compared to the permeability of lipid bilayers to $\mathrm{H}^{+}$and $\mathrm{H}_{2} \mathrm{O}\left(\sim 10^{-4} \mathrm{~m} / \mathrm{s}\right)$ and to $\mathrm{K}^{+}\left(\sim 10^{-12} \mathrm{~m} / \mathrm{s}\right) .{ }^{26}$ Because ionic surfactants such as CTAC do not form free-standing membranes, measuring $\alpha$ could provide an original method for determining their permeability.

\section{Supporting Information Available}

This material is available free of charge at http://pubs.acs.org/.

- Numerical solution of the growth equation.

- Calculation of the form factor of the nanoparticles.

- TR-SAXS fit details. 
- TEM images and analysis.

- AS spectra.

\section{Acknowledgement}

The SOLEIL synchrotron is acknowledged for the provision of beamtime (experiment 20170143) and Javier Perez for his support.

\section{References}

(1) Toshima, N.; Yonezawa, T. Bimetallic Nanoparticles-Novel Materials for Chemical and Physical Applications. New Journal of Chemistry 1998, 22, 1179-1201.

(2) Xia, Y.; Gilroy, K. D.; Peng, H.-C.; Xia, X. Seed-Mediated Growth of Colloidal Metal Nanocrystals. Angewandte Chemie International Edition 2017, 56, 60-95.

(3) Okuno, Y.; Nishioka, K.; Kiya, A.; Nakashima, N.; Ishibashi, A.; Niidome, Y. Uniform and controllable preparation of $\mathrm{Au}-\mathrm{Ag}$ core-shell nanorods using anisotropic silver shell formation on gold nanorods. Nanoscale 2010, 2, 1489-1493.

(4) Park, K.; Drummy, L. F.; Vaia, R. A. Ag shell morphology on Au nanorod core: role of Ag precursor complex. Journal of Materials Chemistry 2011, 21, 15608.

(5) Tebbe, M.; Kuttner, C.; Mayer, M.; Maennel, M.; Pazos-Perez, N.; König, T. A.; Fery, A. Silver-Overgrowth-Induced Changes in Intrinsic Optical Properties of Gold Nanorods: From Noninvasive Monitoring of Growth Kinetics to Tailoring Internal Mirror Charges. The Journal of Physical Chemistry C 2015, 119, 9513-9523.

(6) Hutzler, A.; Schmutzler, T.; Jank, M. P. M.; Branscheid, R.; Unruh, T.; Spiecker, E.; Frey, L. Unravelling the Mechanisms of Gold-Silver Core-Shell Nanostructure Forma- 
tion by in Situ TEM Using an Advanced Liquid Cell Design. Nano Letters 2018, 18, $7222-7229$.

(7) Aliyah, K.; Lyu, J.; Goldmann, C.; Bizien, T.; Hamon, C.; Alloyeau, D.; Constantin, D. Real-Time In Situ Observations Reveal a Double Role for Ascorbic Acid in the Anisotropic Growth of Silver on Gold. The Journal of Physical Chemistry Letters 2020, 11, 2830-2837.

(8) Abécassis, B.; Testard, F.; Spalla, O.; Barboux, P. Probing in situ the Nucleation and Growth of Gold Nanoparticles by Small-Angle X-ray Scattering. Nano Letters 2007, 7, $1723-1727$.

(9) Abécassis, B.; Testard, F.; Kong, Q.; Francois, B.; Spalla, O. Influence of Monomer Feeding on a Fast Gold Nanoparticles Synthesis: Time-Resolved XANES and SAXS Experiments. Langmuir 2010, 26, 13847-13854.

(10) Polte, J.; Erler, R.; Thünemann, A. F.; Sokolov, S.; Ahner, T. T.; Rademann, K.; Emmerling, F.; Kraehnert, R. Nucleation and Growth of Gold Nanoparticles Studied via in situ Small Angle X-ray Scattering at Millisecond Time Resolution. ACS Nano 2010, 4, 1076-1082.

(11) Polte, J.; Ahner, T. T.; Delissen, F.; Sokolov, S.; Emmerling, F.; Thünemann, A. F.; Kraehnert, R. Mechanism of Gold Nanoparticle Formation in the Classical Citrate Synthesis Method Derived from Coupled In Situ XANES and SAXS Evaluation. Journal of the American Chemical Society 2010, 132, 1296-1301.

(12) Koerner, H.; MacCuspie, R. I.; Park, K.; Vaia, R. A. In Situ UV/Vis, SAXS, and TEM Study of Single-Phase Gold Nanoparticle Growth. Chemistry of Materials 2012, 24, 981-995.

(13) Chen, X.; Schröder, J.; Hauschild, S.; Rosenfeldt, S.; Dulle, M.; Förster, S. Simultaneous 
SAXS/WAXS/UV-Vis Study of the Nucleation and Growth of Nanoparticles: A Test of Classical Nucleation Theory. Langmuir 2015, 31, 11678-11691.

(14) Henkel, A.; Schubert, O.; Plech, A.; Sönnichsen, C. Growth Kinetic of a Rod-Shaped Metal Nanocrystal. The Journal of Physical Chemistry C 2009, 113, 10390-10394.

(15) Morita, T.; Tanaka, E.; Inagaki, Y.; Hotta, H.; Shingai, R.; Hatakeyama, Y.; Nishikawa, K.; Murai, H.; Nakano, H.; Hino, K. Aspect-Ratio Dependence on Formation Process of Gold Nanorods Studied by Time-Resolved Distance Distribution Functions. The Journal of Physical Chemistry C 2010, 114, 3804-3810.

(16) Hubert, F.; Testard, F.; Thill, A.; Kong, Q.; Tache, O.; Spalla, O. Growth and Overgrowth of Concentrated Gold Nanorods: Time Resolved SAXS and XANES. Crystal Growth \& Design 2012, 12, 1548-1555.

(17) Carbó-Argibay, E.; Rodríguez-González, B.; Gómez-Graña, S.; Guerrero-Martínez, A.; Pastoriza-Santos, I.; Pérez-Juste, J.; Liz-Marzán, L. M. The Crystalline Structure of Gold Nanorods Revisited: Evidence for Higher-Index Lateral Facets. Angewandte Chemie International Edition 2010, 49, 9397-9400.

(18) Goris, B.; Bals, S.; Van den Broek, W.; Carbó-Argibay, E.; Gómez-Graña, S.; LizMarzán, L. M.; Van Tendeloo, G. Atomic-Scale Determination of Surface Facets in Gold Nanorods. Nature Materials 2012, 11, 930-935.

(19) Gómez-Graña, S.; Goris, B.; Altantzis, T.; Fernández-López, C.; Carbó-Argibay, E.; Guerrero-Martínez, A.; Almora-Barrios, N.; López, N.; Pastoriza-Santos, I.; PérezJuste, J. et al. Au@Ag Nanoparticles: Halides Stabilize $\{100\}$ Facets. The Journal of Physical Chemistry Letters 2013, 4, 2209-2216.

(20) Hamon, C.; Goldmann, C.; Constantin, D. Controlling the Symmetry of Supercrystals Formed by Plasmonic Core-Shell Nanorods with Tunable Cross-Section. Nanoscale 2018, 10, 18362-18369. 
(21) Scarabelli, L.; Grzelczak, M.; Liz-Marzán, L. M. Tuning Gold Nanorod Synthesis through Prereduction with Salicylic Acid. Chemistry of Materials 2013, 25, 4232-4238.

(22) Ye, X.; Jin, L.; Caglayan, H.; Chen, J.; Xing, G.; Zheng, C.; Doan-Nguyen, V.; Kang, Y.; Engheta, N.; Kagan, C. R. et al. Improved Size-Tunable Synthesis of Monodisperse Gold Nanorods through the Use of Aromatic Additives. ACS Nano 2012, 6, 2804-2817.

(23) Kline, S. R. Reduction and analysis of SANS and USANS data using IGOR Pro. Journal of Applied Crystallography 2006, 39, 895-900.

(24) Berthoud, A. Théorie de La Formation Des Faces d'un Cristal. Journal de Chimie Physique 1912, 10, 624-635.

(25) Mersmann, A., Ed. Crystallization Technology Handbook, 2nd ed.; Marcel Dekker: New York, 2001; Chapter 3.

(26) Paula, S.; Volkov, A.; Van Hoek, A.; Haines, T.; Deamer, D. Permeation of Protons, Potassium Ions, and Small Polar Molecules through Phospholipid Bilayers as a Function of Membrane Thickness. Biophysical Journal 1996, 70, 339-348. 


\section{Graphical TOC Entry}

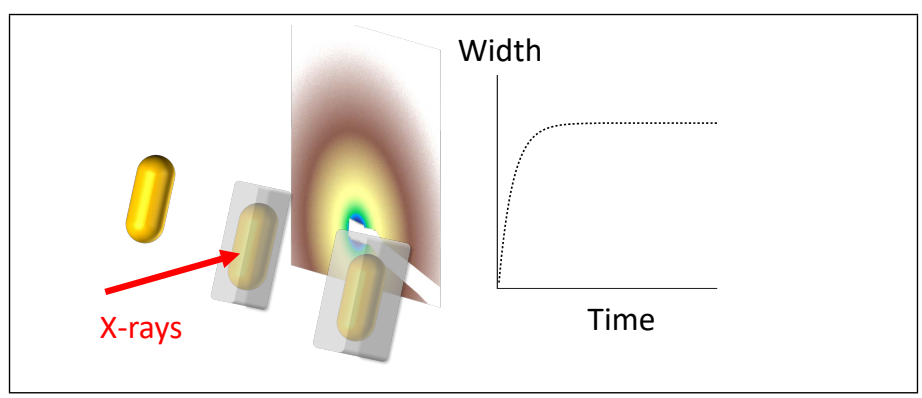

TOC Graphic 